\title{
Image-based Reconstruction of Wire Art
}

\author{
LINGJIE LIU, University of Hong Kong, University College London \\ DUYGU CEYLAN, Adobe Research \\ CHENG LIN, University of Hong Kong \\ WENPING WANG, University of Hong Kong \\ NILOY J. MITRA, University College London
}

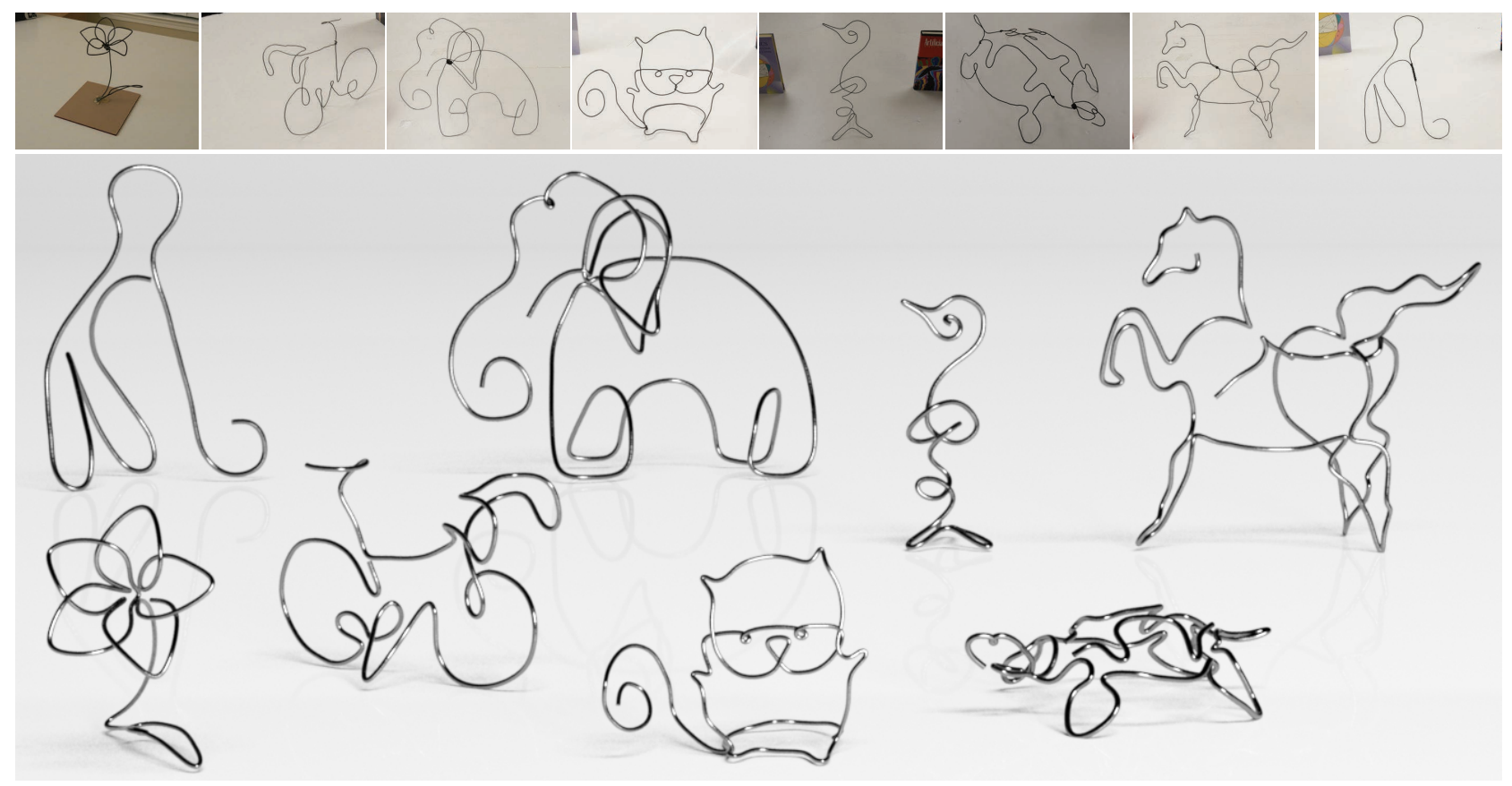

Fig. 1. We present an image-based method to reconstruct wire objects from a few input images ( 3 views in our experiments). Our reconstructions faithfully capture both the $3 \mathrm{D}$ geometry and the topology of the wires.

Objects created by connecting and bending wires are common in furniture design, metal sculpting, wire jewelery, etc. Reconstructing such objects with traditional depth and image based methods is extremely difficult due to their unique characteristics such as lack of features, thin elements, and severe self-occlusions. We present a novel image-based method that reconstructs a set of continuous 3D wires used to create such an object, where each wire is composed of an ordered set of 3D curve segments. Our method exploits two main observations: simplicity - wire objects are often created using only a small number of wires, and smoothness - each wire is primarily smoothly bent with sharp features appearing only at joints or isolated points. In light of these observations, we tackle the challenging image correspondence problem across featureless wires by first generating multiple candidate $3 \mathrm{D}$ curve segments and then solving a global selection problem that balances between image and smoothness cues to identify the correct 3D curves. Next, we recover a decomposition of such curves into a set of distinct and continuous wires by formulating a multiple traveling salesman problem, which finds smooth paths, i.e., wires, connecting the curves. We demonstrate our method

(c) 2017 ACM. This is the author's version of the work. It is posted here for your personal use. Not for redistribution. The definitive Version of Record was published in https://doi.org/10.1145/nnnnnnn.nnnnnnn. on a wide set of real examples with varying complexity and present highfidelity results using only 3 images for each object.

CCS Concepts: • Computing methodologies $\rightarrow$ 3D imaging; Reconstruction; Parametric curve and surface models;

Additional Key Words and Phrases: delicate structure, wire art, image-based reconstruction

\section{ACM Reference format:}

Lingjie Liu, Duygu Ceylan, Cheng Lin, Wenping Wang, and Niloy J. Mitra. 2017. Image-based Reconstruction of Wire Art. 1, 1, Article 1 (August 2017), 11 pages.

DOI: 10.1145/nnnnnnn.nnnnnnn

\section{INTRODUCTION}

Wire art is an ancient art form that refers to creation of complex shapes by bending and connecting wires. First introduced by the Egyptians around 3000 BC (Ogden 1991), wire art is today practiced both by expert designers and hobbyists and is common in furniture design, metal sculptures, jewellery making, etc. Unlike other surface-based objects, wire-based compositions are fundamentally 


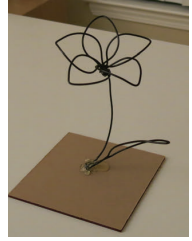

wire object

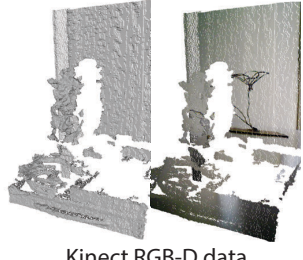

Kinect RGB-D data

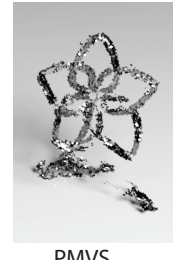

PMVS

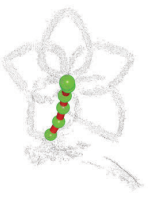

L1-Axis
Fig. 2. While commodity depth sensors, such as the Microsoft Kinect, fail to provide reliable depth data for wire objects (notice how most of the flower is perceived to be planar), PMVS (Furukawa and Ponce 2010), a state-of-the-art multi-view stereo (MVS) method, generates a noisy and partial point cloud. Curve skeleton extraction methods (L1 medial axis (Huang et al. 2013)) are limited when applied to such MVS output.

different as they consist entirely of 1D elements with 'surfaces' being perceptually filled in. This renders such objects more intriguing and creates a desire to digitize them to enrich virtual worlds.

Digitizing wiry objects remains a challenging problem, even though a multitude of depth and image based reconstruction methods exist. Such objects are composed of multiple thin wire structures that occlude each other and are thus difficult to scan even with highend scanners. Moreover, the resolution of commodity depth sensors, such as the Microsoft Kinect, is insufficient to produce reliable depth data for thin features (see Figure 2). Therefore, we resort to imagebased methods which provide higher resolution at a low-cost and a flexible acquisition process. While significant advances have been made in the domain of image based methods, there are two fundamental problems in reconstructing wiry objects that we address in this work.

Correspondence problem. The success of any image based reconstruction method depends on detecting reliable correspondences, i.e., identifying points across images that correspond to the same $3 \mathrm{D}$ point in the physical world. The correspondence problem is especially challenging in the case of wiry objects due to the lack of distinctive image features along thin wires of uniform color. As a result, traditional multi-view stereo (MVS) approaches suffer from significant noise and missing data (see Figure 2). Moreover, the resulting reconstructions have only a low level representation, i.e., point clouds, without a global curve topology required for a faithful representation. Although there exist methods to extract curve skeletons from an input point cloud (Huang et al. 2013), the amount of noise in MVS output renders such methods impractical as shown in Figure 2.

We side-step from the issues of point-level correspondences by utilizing high order curve primitives for image matching. In the absence of distinctive image features, we rely on epipolar cues to find matches among 2D curve segments detected in each input view. For each $2 \mathrm{D}$ curve segment detected in a reference view, there are potentially multiple $2 \mathrm{D}$ curve segments in another view that satisfy the epipolar constraints (Hartley and Zisserman 2004). The number of conforming candidate matches is especially high for wiry objects, which lack surface patches to hide the wires that are in the background. In other words, curves at various depths are simultaneously visible in each image and so result in prohibitively many candidate matches (in our experiments, for each 2D curve segment, we typically get 3-4 candidate matches). While it is possible to locally choose one of these candidate matches based on a data cost, e.g., by measuring the reprojection error of each selected 3D curve segment to the input images, we observe that this is not sufficient to resolve all the ambiguities (see Figure 7). Instead we propose a strategy that explores an additional smoothness cost that favors consecutive 3D curve segments with smooth angles and small gaps. We formulate a global selection problem to choose the desired $3 \mathrm{D}$ curve segments from a set of candidate 3D curve segments by balancing between the data and smoothness costs.

Wire decomposition. Once we construct a set of 3D curve segments, the next critical step is to recover the global topology of these segments and obtain a decomposition of the input object into a set of continuous wires. We observe that objects made of many pieces of wires are often not stable and difficult to assemble, especially for hobbyists. On the other hand, bending only a single piece of wire to create sharp edges is also hard. In light of these observations, we define a graph where each (estimated) 3D curve segment is represented by a vertex and vertices are linked by edges that are assigned costs based on the angle and the distance between the curves they connect. We then formulate an instance of the multiple traveling salesman problem over this graph to find low-cost path(s) connecting all the graph vertices, where each vertex is covered by exactly one path. By associating a cost to each introduced new path, we balance between smoothness (i.e., low cost paths) and simplicity (i.e., as few paths as possible).

Having discovered the global curve topology, we perform a final refinement step to fit a cubic B-spline to each 3D wire. We optimize for the parameters of the splines so that consecutive curve segments along a path are smoothly connected and the reprojection error to the input images is minimized. This refinement step helps to fill small gaps along paths caused by insufficient image information and results in high fidelity reconstructions as shown in Figure 1. Such reconstructions enable easy manipulation or recreation using wire bending machines (Miguel et al. 2016).

We evaluate our method on a variety of real and synthetic examples composed of both single and multiple wires. We also provide comparisons with general purpose reconstruction methods and demonstrate superior results (see Figure 8 and Section 6). Our main contribution is a practical and effective method that reconstructs wiry objects from a very few input images $(3$ in all the presented examples). To our knowledge, no previous method can reconstruct wire sculptures at the complexity of our examples. Our method makes this possible by utilizing high order curve primitives and exploring global (i.e., smoothness and simplicity) as well as local data cues.

\section{RELATED WORK}

Our work builds up on previous methods related to curve-based structure-from-motion and multi-view stereo as well as modeling of delicate structures from images and point clouds.

Modeling of delicate structures. With the many advances in acquisition technologies (e.g., structured light, LiDAR, and more recently commodity depth sensors), we have seen substantial progress in the area of surface reconstruction (Berger et al. 2014) to extract 
high fidelity surfaces from input data. An equally important problem is to improve the acquisition experience in terms of data coverage (Yan et al. 2014), quality (Wu et al. 2014), and speed (Fan et al. 2016). These methods, however, focus on objects with smooth surfaces while scanning thin wires remains challenging due to the limited sensor resolution. In the specific case of objects with delicate structures, several automatic and interactive modeling methods have been presented. Li et al. (2010) introduce the deformable model arterial snakes to reconstruct such objects from high-quality dense $3 \mathrm{D}$ scans. An alternative approach is to fit generalized cylinders to image data (Chen et al. 2013) or point clouds (Yin et al. 2014) where the fitting process is guided by a skeleton either defined manually as in 3-Sweep (Chen et al. 2013) or extracted automatically (Huang et al. 2013). However, thet fact that wire objects do not have sufficient thickness makes it extremely challenging for a user to define a section plane or a non-planar extrusion curve in a 2D image manually. Furthermore, 3D point data (e.g., obtained by MVS algorithms) is not reliable enough to automatically detect curve skeletons as we show in our evaluations. Martin et al. (2014) present a method to reconstruct thin tubular structures from a dense set of images using physics-based simulation of rods to improve accuracy. They use a 3D occupancy grid to disambiguate 2D crossings of cables. However, unlike the clean distribution of occupied cells obtained for cable structures, for typical wiry objects such a grid is very noisy: due to lack of surface occlusion, wires from the front and back of an object are simultaneously visible in each view and this results in ambiguities. Our method recovers the topology of the wires in this challenging case by using a global optimization.

More recently, some approaches to designing wire objects have been proposed with a specific focus on fabricating these designs. Iarussi et al. (2015) tackle the problem of extracting the topology of a wire jewelry from a single drawing. However, their method assumes that the curves are planar and fully visible in the single image input. Miguel et al. (2016) present an interactive system to convert a closed surface mesh to a self-supporting wire sculpture. While both approaches explore similar characteristics of wire objects, e.g., simplicity, they fundamentally focus on a different problem of bringing digital designs into life. Thus, our approach is complementary and can provide input to these methods.

Curve-based Structure-from-Motion. In recent years, a multitude of successful structure-from-motion (SfM) (Snavely et al. 2006; Wu et al. 2011a) and multi-view stereo (MVS) (Furukawa and Ponce 2010; Goesele et al. 2007) algorithms have been developed. These methods work on the general principle of identifying point correspondences across images that represent the same world-space surface locations. While these methods produce impressive results for objects with reliable texture information, they perform poorly when there are insufficient discriminative point features. Some researchers have tackled this limitation by exploring the presence of higher order features such as lines (Baillard et al. 1999; Hofer et al. 2014, 2013; Jain et al. 2010) and curves (Xiao and Li 2005). We refer the reader to the recent work of Fabbri et al. (2016) for a theoretical foundation of the multi-view geometry properties of curves.

Curve-based reconstruction methods can be grouped according to the simplifying assumptions they make: Berthilsson et al. (2001) assume that each 3D curve is fully visible in each view with no selfocclusions; Hong et al. (2004) focus on reflective symmetric curves that are fully visible in one or two images; assuming access to the range of depth values in the reconstructed scene, Kahl and August (2003) assign depth values to 2D curves, while Teney and Piater (2012) design a probability distribution to model the space of plausible curve reconstructions. Rao et al. (2012) explore known correspondences between endpoints of planar curves to develop a curve-based SLAM (simultaneous localization and mapping) system. Similarly, Nurutdinova et al. (2015) assume that image curves are already in correspondence and optimize for both camera calibration and parameters of the reconstructed space curves.

In the absence of prior knowledge, a typical workflow, which we also adopt, is to first detect $2 \mathrm{D}$ curve segments in each image and then utilize epipolar constraints to generate hypotheses of corresponding curves across image pairs. This process typically results in multiple ambiguous matches and an additional view is required for verification (Robert and Faugeras 1991). Recent work (Fabbri and Kimia 2010; Usumezbas et al. 2016) uses this workflow to reconstruct curvilinear structures in general scenes requiring a dense set of images. Our work, in contrast, reconstructs wireframe objects from as few as three input views.

All the above mentioned previous works obtain a reconstruction in the form of individual curve segments, which possibly suffers from noisy measurements and gaps. Instead, we globally extract continuous curve paths to produce more faithful representations of wire objects. A similar path finding approach has been proposed in the context of medical imaging (Delmas et al. 2015); however, no previous approach has demonstrated results at the complexity of the examples we show.

\section{OVERVIEW}

The input to our algorithm is a set of $n$ (in our experiments $n=$ 3) images, $\mathcal{I}=\left\{\mathcal{I}^{1}, \ldots, \mathcal{I}^{n}\right\}$, of a $3 \mathrm{D}$ wire model captured from different viewpoints along with their camera parameters, $\mathcal{K}=$ $\left\{\mathcal{K}^{1}, \ldots, \mathcal{K}^{n}\right\}$. The output of our method is a set of $k$ reconstructed $3 \mathrm{D}$ wires $\mathcal{W}=\left\{\mathcal{W}_{1}, \ldots, \mathcal{W}_{k}\right\}$, where each wire is a continuous path represented as a B-spline curve. We provide an overview of this pipeline in Figure 3.

In a pre-processing stage, we extract a set of $2 \mathrm{D}$ curve segments in each image. Specifically, we first convert the images to the $\mathrm{YCbCr}$ color space with histogram equalization on Y-channel (luminance component) to increase the contrast of the wire regions relative to the image background. Then, starting from an arbitrary pixel in the perceived wire region, we utilize a flooding method to extract all the connected pixels that have a similar color and apply a thinning algorithm to extract a one-pixel wide curve. Finally, we decompose each such curve into segments such that no curve segment contains any joint or branching point (Kovesi 2017). As a result, for each image $\mathcal{I}^{j}$, we obtain a set of 2D curve segments denoted as $\mathbf{c}^{\mathbf{j}}=\left\{c_{i}^{j}\right\}$.

Given a set of $2 \mathrm{D}$ curves extracted from each image, our first goal is to generate a corresponding set of candidate $3 \mathrm{D}$ curve segments. To accomplish this goal, we establish continuos correspondences between matching 2D curve segments from different images. 


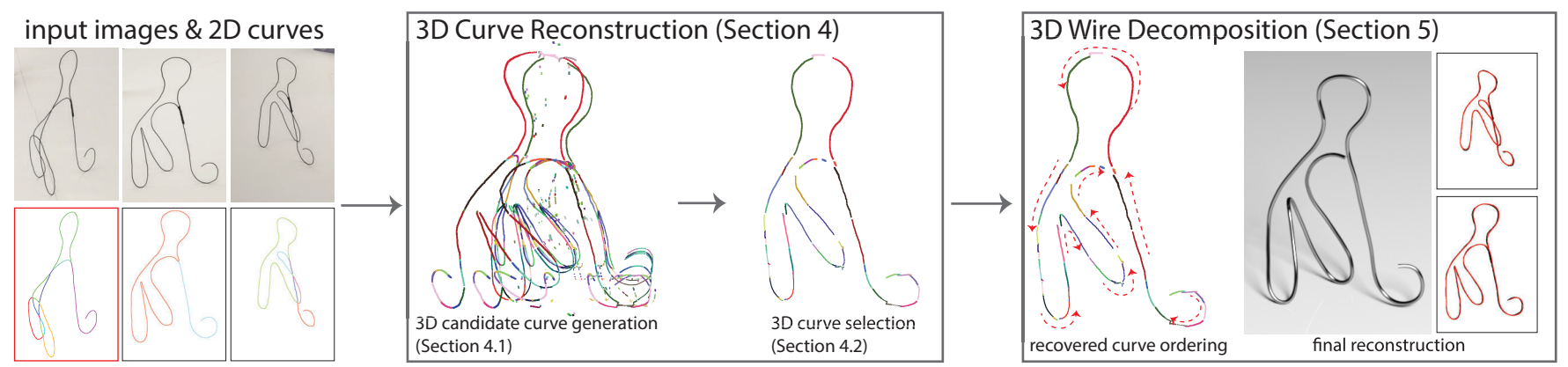

Fig. 3. Given a set of input images of a wire object, our method first detects 2D curve segments in each image. We then generate a set of candidate 3D curve segments corresponding to each 2D image curve (Section 4.1) and solve a global selection problem to choose one 3D curve from each candidate set (Section 4.2). Finally, we recover the global topology of the 3D curve segments (as denoted by the dashed red arrows) and decompose the object into a set of continuous wires (Section 5). We show this final reconstruction both in 3D and also how its projection (in red) aligns with the original image curves (in black).

We use a conservative strategy when decomposing $2 \mathrm{D}$ curves in each image into segments free of branching points to ensure that no 2D curve segment contains projections of disjoint $3 \mathrm{D}$ curves. The red highlighted region in the

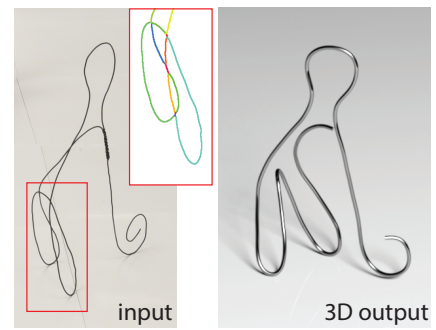
inset figure shows an example, where each 2D curve segment is denoted with a different color. Some of the branching points detected in the images may have been created due to self-occlusions and separate $2 \mathrm{D}$ curve segments that are projections of a single continuous 3D curve. While this conservative decomposition strategy results in the loss of such continuity relations, they are recovered in the subsequent stages of our algorithm. For example, the final 3D result shown in the inset consists of a single continuous wire.

For each 2D curve segment $c_{i}^{j}$ detected in a reference view $I^{j}$, we generate a set of candidate 3D curve segments by establishing correspondences with 2D curve segments in a neighboring view that satisfy the epipolar constraints (Section 4.1). Once such sets of candidate 3D curve segments are generated for all views, we formulate a global selection problem that solves a quadratic optimization problem to choose one 3D curve segment from each candidate set (Section 4.2). This optimization not only minimizes the re-projection error of a selected 3D curve segment with respect to each view but also favors consecutive 3D curve segments that form smooth angles. As a result, we select a set of $3 \mathrm{D}$ curve segments, denoted as $C_{\text {opt }}$.

Next, to extract a global curve topology among the individual and disjoint $3 \mathrm{D}$ curve segments in $C_{\text {opt }}$, we solve a multiple traveling salesman problem (mTSP) to identify continuous and smooth paths such that each 3D curve segment is contained in exactly one path (Section 5). Each such path corresponds to a continuous wire composed of a sequence of 3D curve segments which are a subset of $C_{\text {opt }}$. Finally, for a smooth and compact representation, we represent each wire as a smooth cubic B-spline curve and optimize for its parameters using a fitting procedure to minimize its projection error with respect to the input images.

Next, we describe the respective steps in detail.

\section{3D CURVE RECONSTRUCTION}

The 3D curve reconstruction step of our method first generates a set of candidate 3D curve segments and then solves a global selection optimization to identify the correct 3D curves among these candidates. Without loss of generality, we first explain this process for a given pair of reference and neighboring views $\left(\mathcal{I}^{r}, \mathcal{I}^{n}\right)$ and then describe how it can be easily extended to multiple views.

\subsection{D Candidate Curve Generation}

Given a set of curve segments $\mathbf{c}^{\mathbf{r}}=\left\{c_{i}^{r}\right\}$ detected in a reference view $\mathcal{I}^{r}$, we represent each $c_{i}^{r}$ as a sequence of densely sampled image points, $p_{i, j}^{r}, j=1,2, \ldots, s$. For each $c_{i}^{r}$, starting from the first sample point $p_{i, 1}^{r}$, we compute its corresponding epipolar line, $l_{i, 1}^{r \rightarrow n}$, in a neighboring view $\mathcal{I}^{n}$. Each epipolar line $l_{i, 1}^{r \rightarrow n}$ is likely to intersect multiple disjoint $2 \mathrm{D}$ curve segments in $\mathcal{I}^{n}$, each being a possible match for $c_{i}^{r}$ (see Figure 4) ${ }^{1}$. We expect the epipolar lines corresponding to consecutive sample points $p_{i, j}^{r}$ to result in intersections with the same set of candidate matching curve segments due to the

\footnotetext{
${ }^{1}$ We note that there may be degenerate cases where an epipolar line is locally almost parallel to a $2 \mathrm{D}$ curve segment making it difficult to compute robust intersections. We refer the reader to the Appendix for a discussion of how we handle these cases.
}
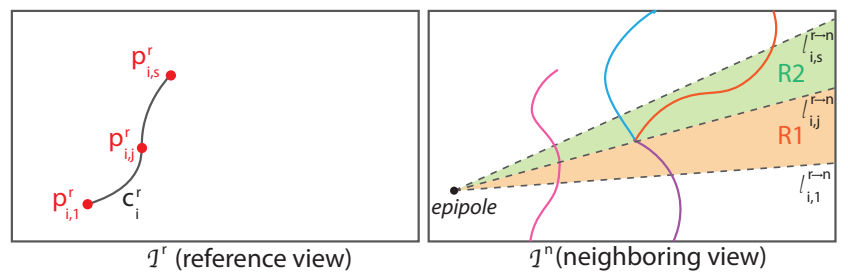

Fig. 4. We show a 2D curve segment $c_{i}^{r}$ in $\mathcal{I}^{r}$ along with sample points $\left(p_{i, 1}^{r}\right.$, $\left.p_{i, j}^{r}, p_{i, s}^{r}\right)$ and their corresponding epipolar lines $\left(l_{i, 1}^{r \rightarrow n}, l_{i, j}^{r \rightarrow n}, l_{i, s}^{r \rightarrow n}\right)$ in $\mathcal{I}^{n}$. Epipolar lines corresponding to sample points between $p_{i, 1}^{r}$ and $p_{i, j}^{r}$ result in two intersection points in $\mathcal{I}^{n}$ (R1) and generate two candidate matching 2D curve segments (pink and purple). Starting with $p_{i, j}^{r}$, epipolar lines result in three intersection points (R2). Thus we break $c_{i}^{r}$ into two segments at $p_{i, j}^{r}$ such that for the segment between $p_{i, j}^{r}$ and $p_{i, s}^{r}$ three candidate matching 2D curve segments (pink, blue, and orange) are generated. 


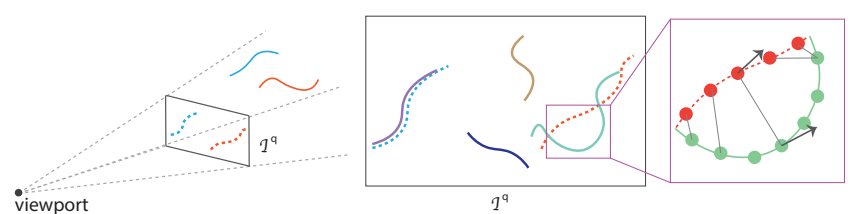

Fig. 5. Given a candidate 3D curve segment, we project it to a third view $\mathcal{I}^{q}$ and find the 2D curve segment in $\mathcal{I}^{q}$ that has the smallest aggregated closest point distance with its projection. We compute a confidence cost for the 3D curve segment which measures both the distance and the angular deviation between such closest points. 3D curve segments (in orange) which receive a high confidence cost can be pruned, while others (in blue) are kept as a candidate.

continuity of image curves. Thus, we trace out these intersections as long as the number of intersections for consecutive sample points stays constant. If we reach a sample point $p_{i, j}^{r}$ on $c_{i}^{r}$ whose epipolar line intersects a different number of $2 \mathrm{D}$ curve segments in $\mathcal{I}^{n}$ (e.g., due to a discontinuity resulting from self-occlusions), we break $c_{i}^{r}$ into two segments consisting of sample points $\left\{p_{i, 1}^{r}, p_{i, 2}^{r}, \ldots, p_{i, j-1}^{r}\right\}$ and $\left\{p_{i, j}^{r}, p_{i, j+1}^{r}, \ldots, p_{i, s}^{r}\right\}$, respectively. By repeatedly tracing the intersection points of the consecutive epipolar lines and splitting $c_{i}^{r}$ as necessary, we obtain a resulting set of 2D curve segments $\mathbf{c}^{\mathrm{r}}$ in $\mathcal{I}^{r}$, where for each $c_{i}^{r}$ we have a set of matching candidate 2D curve segments $c_{i, j}^{r \rightarrow n}, j=1,2, \ldots, m$ in the neighboring view $\mathcal{I}^{n}$.

Given a curve segment $c_{i}^{r}$ in $\mathcal{I}^{r}$ and its candidate matching curve segments $c_{i, j}^{r \rightarrow n}$ in $\mathcal{I}^{n}, j=1,2, \ldots, m$, we generate a set of 3D curve segments $C_{i, j}^{r}, j=1,2, \ldots, m$, each represented by a sequence of $3 \mathrm{D}$ points reconstructed by triangulating the corresponding sample points in $\mathcal{I}^{r}$ and $\mathcal{I}^{n}$. Each $C_{i, j}^{r}$ is potentially the correct 3D curve segment for $c_{i}^{r} \in \mathcal{I}^{r}$ and is also compatible with $\mathcal{I}^{n}$ by construction. We use a third view $\mathcal{I}^{q}$ to help asses the likelihood of each of $C_{i, j}^{r}$ to be the correct $3 \mathrm{D}$ curve segment corresponding to $c_{i}^{r}$ (see Figure 5)

In particular, we project each $C_{i, j}^{r}$ to $\mathcal{I}^{q}$ and find the 2D curve segment in $\mathcal{I}^{q}$ that is closest to its projection. We compute this closest $2 \mathrm{D}$ curve segment by establishing closest point correspondences between each sample on the projection of $C_{i, j}^{r}$ and the sample points on each $c_{o}^{q} \in \mathcal{I}^{q}$ and choosing the $c_{o}^{q}$ that results in the smallest aggregated distance between such correspondences. Given a set of point correspondences $(u, v)$, where $u$ lies along the projection of the 3D curve segment $C_{i, j}^{r}$ to $\mathcal{I}^{q}$ and $v$ is its closest point correspondence on the $2 \mathrm{D}$ curve segment $c_{o}^{q} \in \mathcal{I}^{q}$, we compute a confidence $\operatorname{cost}, \mathcal{S}\left(C_{i, j}^{r}\right)$, as:

$$
\mathcal{S}\left(C_{i, j}^{r}\right)=\frac{\sum_{(u, v)}\left(\left(\|u-v\| / \operatorname{diag}\left(\mathcal{I}^{q}\right)\right)+\left(1-\left|t_{u} \cdot t_{v}\right|\right)\right)}{s},
$$

where we measure both the distances between the corresponding points and the deviation between their tangential directions, $\left(t_{u}, t_{v}\right)$ (see Figure 5). We normalize the distance measures by the length of the diagonal of the image, $\operatorname{diag}\left(\mathcal{I}^{q}\right)$, and normalize the aggregated distance and tangential deviation by $s$, the number of sample points on $C_{i, j}^{r}$. This confidence cost is used in the subsequent stage of our method to identify the correct 3D curve segments.

\subsection{D Curve Selection}

In the previous step, for each $2 \mathrm{D}$ curve segment $c_{i}^{r}$, we reconstruct a set of 3D curve segments, $\mathrm{C}_{\mathbf{i}}^{\mathrm{r}}=\left\{C_{i, j}^{r}\right\}$, composed of multiple candidates satisfying the epipolar constraints (see Figure 6) (in our experiments we often have 3-4 candidates for each curve segment). Next, we explore two type of cues to choose one 3D curve segment for each $c_{i}^{r}$ as its true 3D proxy. First, for each $c_{i}^{r}$, we want to select the $3 \mathrm{D}$ curve segment that matches image observations well. However, due to many self-occlusions, image observations alone are not sufficient to resolve all the ambiguities. Therefore, we also explore pairwise relations between 3D curve segments. Since the true $3 \mathrm{D}$ curve segments are assumed to be taken from an object created by bending continuous wires, consecutive curve segments along this wire are smoothly connected. Thus, pairs of nearby 3D curve segments with smooth angles are more likely to exist together, which provides an effective pairwise cue. We formalize these cues in an optimization problem as follows. First, for ease of notation, we denote $\mathcal{A}^{r}=\cup_{i} \mathbf{C}_{\mathrm{i}}^{\mathrm{r}}$, the union of all candidate 3D curve segment sets $\mathrm{C}_{\mathrm{i}}^{\mathrm{r}}$. For each $3 \mathrm{D}$ curve segment in $\mathcal{A}_{j} \in \mathcal{A}^{r}$, we define a binary indicator variable $x_{j}$ where $x_{j}=1$ if the corresponding curve segment is selected and $x_{j}=0$ otherwise. We denote by $\mathrm{X}$ the vector formed by concatenating all such binary variables. Since, our goal
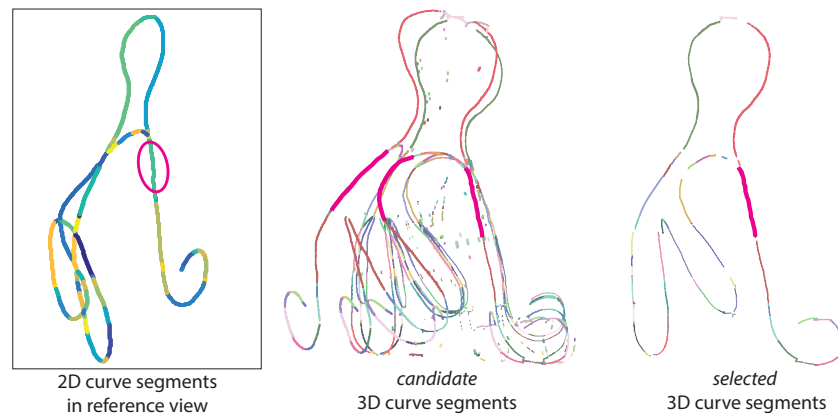

Fig. 6. After the $3 \mathrm{D}$ candidate curve generation step, for each $2 \mathrm{D}$ curve segment we obtain multiple candidates (e.g., for the highlighted 2D curve segment, we obtain 3 candidate curves in $3 \mathrm{D}$ (in pink)). The 3D curve selection process identifies the correct 3D curve among such candidates solving a global selection problem.

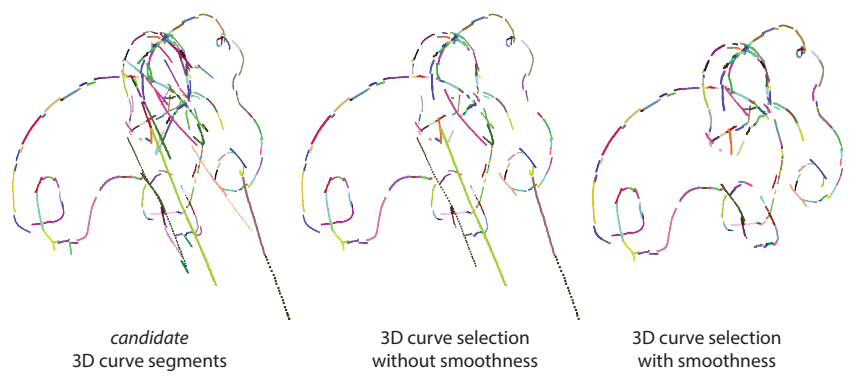

Fig. 7. For a given set of candidate 3D curve segments, using only the unary data term results in the selection of many wrong 3D curve segments. This shows the necessity of a global optimization with additional smoothness priors. 
is to select only one 3D curve segment from each candidate set, we define the linear constraint, $\sum \mathcal{A}_{j} \in \mathbf{C}_{\mathbf{i}}^{\mathbf{r}} \mathbf{X}(j)=1$ for each $\mathbf{C}_{\mathbf{i}}^{\mathbf{r}}$.

We evaluate the compatibility of each 3D curve segment using the confidence cost defined in Equation 1. We concatenate the confidence scores of all the $3 \mathrm{D}$ curve segments $\mathcal{A}_{j} \in \mathcal{A}^{r}$ in a vector $\mathrm{U}$ such that $\mathrm{U}_{j}=\mathcal{S}\left(\mathcal{A}_{j}\right)$.

Next, in order to evaluate the pairwise relations between 3D curve segments $\mathcal{A}_{i}$ and $\mathcal{A}_{j}$, we define a pairwise cost $d\left(\mathcal{A}_{i}, \mathcal{A}_{j}\right)$ :

$$
d\left(\mathcal{A}_{i}, \mathcal{A}_{j}\right)=e\left(\mathcal{A}_{i}, \mathcal{A}_{j}\right)+\mu f\left(\mathcal{A}_{i}, \mathcal{A}_{j}\right) .
$$

$e\left(\mathcal{A}_{i}, \mathcal{A}_{j}\right)$ measures the distance in 3D between the closest endpoints of $\mathcal{A}_{i}$ and $\mathcal{A}_{j}$ and $f\left(\mathcal{A}_{i}, \mathcal{A}_{j}\right)=(1-\cos \alpha) / 2$, where $\alpha$ is the angle between the tangent directions of these closest endpoints. Here $\mu$ denotes the relative importance between the distance and angle terms ( $\mu=1$ in our experiments). We aggregate all pairwise relations in a square matrix $\mathrm{V}$ of dimension $b \times b$ where $b$ is the cardinality of $\mathcal{A}^{r}$ (i.e., the total number of candidate 3D curve segments). We set $V_{i j}=d\left(\mathcal{A}_{i}, \mathcal{A}_{j}\right)$ for all non-diagonal entries and set the diagonal entries to zero.

Given $\mathrm{U}$ and $\mathrm{V}$, we optimize for $\mathrm{X}^{\star}$ that minimizes the following:

$$
\begin{aligned}
& \mathbf{X}^{\star}: \underset{\mathbf{X}}{\operatorname{argmin}} \mathbf{U}^{T} \mathbf{X}+\lambda \mathbf{X}^{T} \mathbf{V X}, \\
& \text { subject to } \forall \mathbf{C}_{\mathbf{i}}^{\mathbf{r}}: \sum_{\mathcal{A}_{j} \in \mathrm{C}_{\mathbf{i}}^{\mathrm{r}}} \mathbf{X}(j)=1
\end{aligned}
$$

where $\lambda$ determines the relative weighting of the unary and binary cues and is set to 0.1-0.2 in our experiments (based on thickness and size of the object). We solve Equation 3 using the quadratic programming package in Matlab (The MathWorks 2016) and obtain an optimized set of 3D curve segments $C_{\text {opt }}$ which contains exactly one $3 \mathrm{D}$ curve segment as the true $3 \mathrm{D}$ proxy for each $2 \mathrm{D}$ curve segment $c_{i}^{r}$.

While it is possible to solve Equation 3 over all of the candidate 3D curve segments, in cases where this becomes computationally inefficient (due to a very large number of unknowns), it is possible to employ a pruning strategy where 3D curve segments with a confidence cost greater than a threshold (1.5-2 in our experiments) are pre-filtered. Finally, in order to show the importance of utilizing both unary and binary cues, for a given set of candidate 3D curve segments, we solve Equation 3 twice, with and without the binary term. As shown in Figure 7, using only unary terms results in selection of many wrong candidates. This proves the effectiveness of our global selection strategy.

\subsection{Taking Alternative Reference Views}

We have described the 3D curve segment reconstruction step that generates 3D candidate curves from a pair of reference and neighbor views and uses a third view for verification. When there are more views, we utilize an incremental reconstruction strategy. Starting from a pair of reference and neighbor views, we reconstruct a set of $3 \mathrm{D}$ curves for each $2 \mathrm{D}$ curve segment in the reference view as just described. We then choose a new view as reference view and find a compatible neighboring view (a view with a good range of parallax as proposed by Goesele et al. (2007)). We project all the 3D curve segments reconstructed so far to the reference view and mark all (parts of) 2D curve segments that are covered by a projection as processed. A 2D curve segment is considered to be covered by a projection of a 3D curve segment if the aggregated distance and tangent deviation between the closest points among the 2D segment and the projection is below a certain threshold (0.02-0.05\% compared to bounding box diagonal). We perform the same reconstruction step for all the unprocessed 2D curve segments in the new reference view. We repeat this step until all views have been considered as a reference view.

\section{3D WIRE DECOMPOSITION}

Once we have constructed a set of $3 \mathrm{D}$ curve segments, $C_{\text {opt }}$, as explained in the previous section, our next goal is to recover the global topology of these curves and decompose them into a set of distinct continuous wires. To perform this decomposition, we exploit two unique features of wire objects we consider as input. First, such objects are often composed of only a small number of wires because it is not trivial to stably connect many pieces of wires. Second, it is hard to bend wires to create sharp angles due to physical resistance. Therefore, we expect each output wire to be as smooth as possible. In light of these considerations, we formulate the 3D wire decomposition as a multiple traveling salesman problem (mTSP) as described in the following.

Given the set of 3D curve segments, $C_{\text {opt }}=\left\{C_{i}\right\}, i=1,2, \ldots b$, we construct a directed graph $G=(\mathcal{V}, \mathcal{E})$, where each $C_{i}$ is represented as a vertex $V_{i} \in \mathcal{V}$. We define an additional start node, $V_{0}$, such that each path defined over this graph starts and ends at this node. A directed edge $e_{i j} \in \mathcal{E}$ going from the node $V_{i}$ to $V_{j}$ is assigned a cost $w_{i j}$ based on the pairwise relation between the corresponding 3D curve segments $C_{i}$ and $C_{j}$. Precisely, $w_{i j}=d\left(C_{i}, C_{j}\right)$ as given in Equation 2. The edge costs are defined symmetrically, i.e., $w_{i j}=w_{j i}$.

Given $G=(\mathcal{V}, \mathcal{E})$, our goal is to find $k$ distinct paths that start and end at $V_{0}$ such that each $V_{i}(i>0)$ is contained in exactly one path. Each of these paths corresponds to a continuous 3D wire used to create the input wire model. Since each path is uniquely defined by the edges it is composed of, each edge $e_{i j} \in \mathcal{E}$ is assigned a binary variable $x_{i j}: x_{i j}=1$ if $e_{i j}$ is contained in a path and $x_{i j}=0$ otherwise. Furthermore, each node $V_{i} \in \mathcal{V}$ is assigned an auxiliary integer variable $u_{i} \in \mathbb{N}$ to denote the order in which $V_{i}$ is visited along a path, $u_{0}=0$ for the dummy start node $V_{0}$. We formulate our path finding problem as minimizing the total cost of the selected edges and the number of paths:

$$
\left\{k^{\star}, x_{i j}^{\star}\right\}:=\underset{\left\{k, x_{i j}\right\}}{\operatorname{argmin}} \sum_{i, j} x_{i j} w_{i j}+\xi \dot{k} .
$$

$\xi$ is a fixed cost associated with using a unique path. In order to ensure each path is valid (i.e., each vertex is visited by exactly one path and no path is composed of disconnected cycles), Equation 4 is solved subject to the following constraints.

To ensure each node $V_{i}(i>0)$ is visited by a path, exactly one of the incoming and outgoing edges of a node needs to be selected in the solution:

$$
\begin{aligned}
& \forall i>0: \sum_{j, j \neq i} x_{j i}=1, \\
& \forall i>0: \sum_{j, j \neq i} x_{i j}=1 .
\end{aligned}
$$


Each of the $k$ paths is required to start and end at the start node $V_{0}$ :

$$
\begin{gathered}
\sum_{j, j>0} x_{j 0}=k, \\
\sum_{j, j>0} x_{0 j}=k .
\end{gathered}
$$

In order to avoid paths composed of disconnected cycles, subtour elimination constraints as proposed by Kara and Bektas (2006) are defined:

$$
\begin{aligned}
& \forall i>0: \\
& \quad u_{i}+(b-1-k) x_{0 i}-x_{i 0} \leq b-k, \\
& \quad u_{i}+x_{0 i} \geq 2, \\
& \forall i, j>0, i \neq j: \\
& \quad u_{i}-u_{j}+(b-k+1) x_{i j}+(b-1-k) x_{j i} \leq b-k .
\end{aligned}
$$

We use the Gurobi optimization package (Gurobi Optimization 2016) to solve the binary-integer optimization problem given in Equation 4 with respect to the constraints defined in Equations 5, 6, and 7 . As a result, we obtain a set of $k$ continuous wires, $\mathcal{W}=$ $\left\{\mathcal{W}_{1}, \ldots, \mathcal{W}_{k}\right\}$, where each $\mathcal{W}_{i}$ is composed of an ordered set of 3D curve segments.

We note that $\xi$, the cost of using a unique wire, provides a tradeoff in the number of wires included in a decomposition. Increasing this cost prefers decompositions composed of fewer wires. In our experiments, we found setting $\xi=1 / 8^{t h}$ of the maximum edge cost provides a good balance. We also experimented with optimizing Equation 4 with known number of wires, i.e. manually setting $k$. In the special case of $k=1$ (i.e., for objects composed of a single wire), this formulation reduces to the standard travelling salesman problem where the goal is to find a single path that visits each node. Finally, while Equation 4 can be optimized over a fully connected graph $G$, some edges with very high costs can be pre-pruned for better computational efficiency.

\section{Image-guided Curve Smoothing}

At this stage, we have fully recovered all the 3D proxy information we need to represent the input object. Due to instabilities in lifting very small $2 \mathrm{D}$ curve segments to $3 \mathrm{D}$, there may be small gaps between the 3D curve segments along a given wire decomposition. Thus, in a final stage, we represent each wire $\mathcal{W}_{i}$ with a cubic Bspline and optimize for its parameters so that the projection of the resulting 3D wire matches the image observations. In particular given a wire $\mathcal{W}_{i}$, we represent it as:

$$
\mathcal{W}_{i}=\mathcal{B}(t)=\sum_{j=1}^{g} \mathcal{X}_{j} \phi_{j}(t),
$$

where $\mathcal{X}_{j}$ are the control points and $\phi_{j}$ are fixed cubic B-spline basis functions. If the total number of points sampled along all the $3 \mathrm{D}$ curve segments contained in $\mathcal{W}_{i}$ is $h$, we define $g=h / 10$ control points. We first fit a B-spline to the set of all sample points along $\mathcal{W}_{i}$ with each sample point $u_{a}, a=1,2, \ldots h$ associated with a fixed parameter value along this spline, $\mathcal{B}\left(t_{a}\right), 0 \leq t_{a} \leq 1$.

Given this initial B-spline fitting curve in 3D, we next perform an iterative optimization to obtain a refined set of control points $X_{j}^{\star}$ that minimizes the following energy:

$$
\begin{aligned}
& \mathcal{X}_{j}^{\star}:=\underset{\mathcal{X}_{j}}{\operatorname{argmin}} \sum_{a=1}^{h} \sum_{o=1}^{n}\left\|\mathcal{K}^{o}\left(\mathcal{B}\left(t_{a}\right)\right)-p^{o}\right\|^{2}+ \\
& \omega \sum_{a=2}^{h-1}\left\|\mathcal{B}\left(t_{a-1}\right)-2 \mathcal{B}\left(t_{a}\right)+\mathcal{B}\left(t_{a+1}\right)\right\|^{2} .
\end{aligned}
$$

The first term minimizes the distance between $\mathcal{K}^{o}\left(\mathcal{B}\left(t_{a}\right)\right)$, the projection of a 3D sample point $u_{a}$ to image $\mathcal{I}^{o}$, and its closest point correspondence $p^{o} \in \mathcal{I}^{o}$. We project each sample point to the images it is visible in. The second term imposes a Laplacian smoothness penalty between consecutive sample points and $\omega$ (set to $10^{3}$ in our experiments) defines the relative importance between the data and smoothness terms.

We solve Equation 9 in an iterative manner, re-establishing the correspondences between the projections of 3D sample points and $2 \mathrm{D}$ sample points in the images at each iteration. Typically, this process converges in 5-15 iterations and the resulting B-spline representation of each wire is free of gaps and provides an accurate, smooth, and compact reconstruction of the object.

\section{RESULTS}

We evaluate our method on a wide set of examples with varying complexity. For each of these examples we utilize 3 images of the wire object taken from different viewpoints as input, together with their camera parameters. We use the open-source structure-frommotion (SfM) tool, VisualSFM (Wu 2011; Wu et al. 2011b), to obtain the camera parameters in a pre-calibration step. Note that we make use of nearby textured objects to ensure that a sufficient number of image correspondences are detected for reliable SfM computation.

For each of our examples, we provide a sample input image and a rendering of our final reconstruction in Figure 8 and refer to the accompanying video and supplementary material for more closeups. Our examples have varying complexities, each being composed of 1-3 wires. For each of these examples, our method provides a faithful 3D geometry and a plausible wire decomposition. We note that during wire decomposition a specific challenging case is when four curve segments come together (see inset). In this case, there are two possible options for

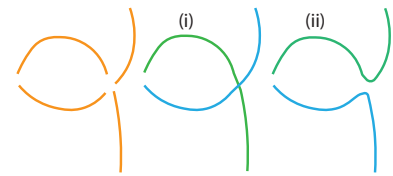
connecting these curves, i.e. (i) a crossing, or (ii) or a continuous path with sharp bends, both resulting in plausible decompositions. Our method prefers smooth crossings since we utilize the smoothness prior. If this is not the desired decomposition, the user may want to reclassify junction points.

\section{Quantitative Evaluations}

In order to quantitatively evaluate our algorithm, we reconstruct a synthetic wire object (similar in complexity to the CAT example) from its renderings using ground truth camera parameters. Furthermore, we generate reconstructions by adding noise to the camera parameters. We represent the rotation of the camera with Euler angles and add random noise to each of the angles sampled from a 
- Liu et al.
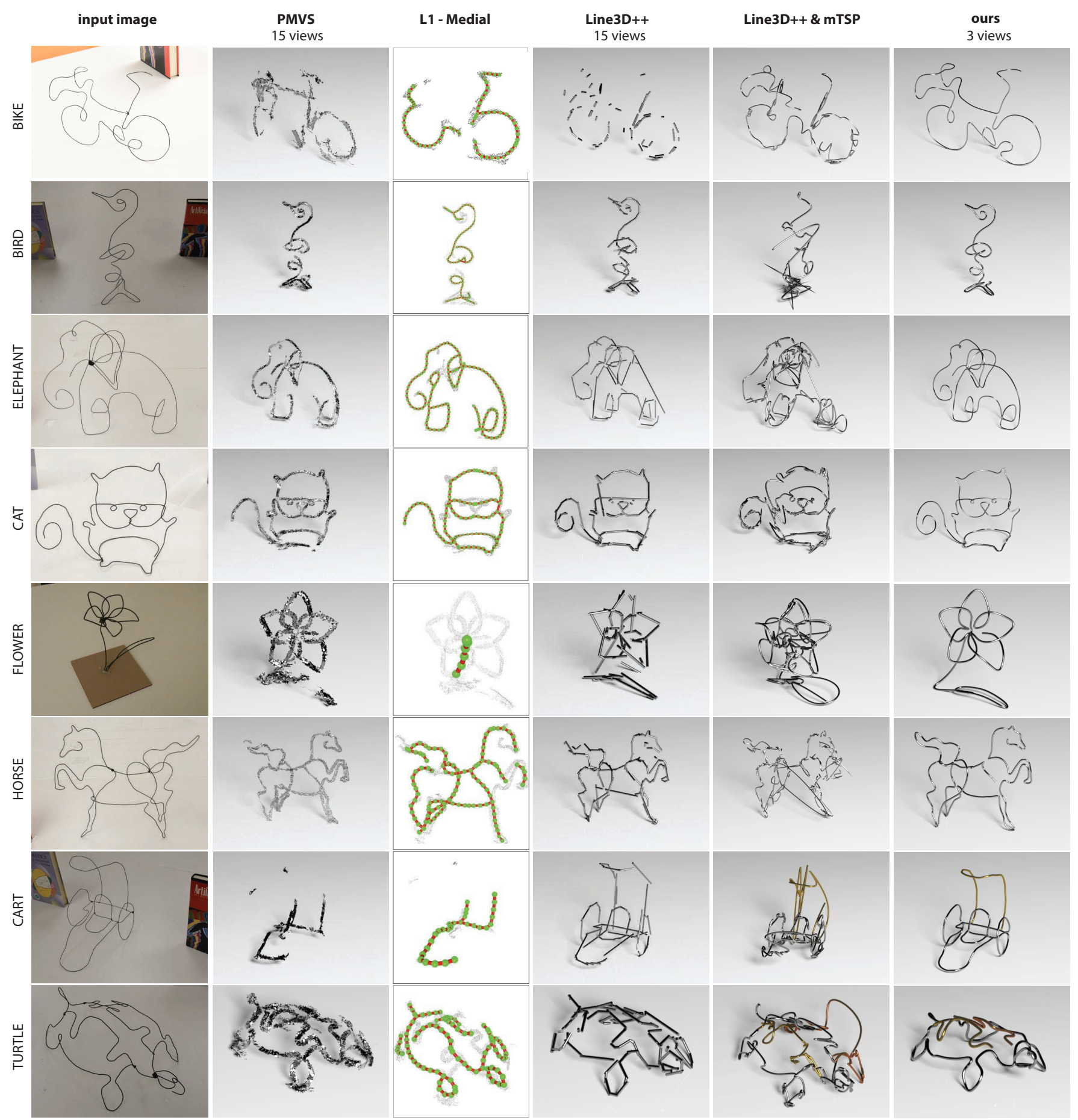

Fig. 8. We evaluate our method on several objects of varying complexity. For each object, we provide one of the input images and our final 3D reconstruction. We also provide comparisons with PMVS (Furukawa and Ponce 2010) and Line3D++ (Hofer et al. 2016) run on a set of input images consisting of 15 views. Finally, we evaluate the L1-medial skeleton extraction method of Huang et al. (2013) on the point clouds generated by PMVS. We also run the mTSP formulation on the output of Line3D++ output to demonstrate how small variations can lead to large topological errors with significant errors in the final output. 
normal distribution with mean zero and standard deviation of one degree. For translation, we add random noise by sampling from a normal distribution of mean zero and a standard deviation of $0.1 \%$, $0.2 \%$, and $0.3 \%$ of the length of the diagonal of the bounding box of the synthetic model in 3D. In each case, we measure the closest point distances between the ground truth and the reconstructed model in 3D. We report the average and maximum of such closest point distances in Table 1. We note that we report the errors as a percentage of the length of the diagonal of the bounding box of synthetic model in 3D.

We also quantitatively evaluate the performance of our approach on real examples. We compute a re-projection error by sampling points on the reconstructed 3D wires, projecting these samples to the input views, and measuring the distance between the projected points and the closest points sampled on the 2D curves for each view. We report the average and maximum of such re-projection errors for each input view in Table 2. We note that we report the errors as a percentage of the length of the diagonal of the $2 \mathrm{D}$ bounding box of the wiry object in the corresponding view.

\section{Performance}

We measure the execution time of different stages of our algorithm on a machine with Intel i7 $3.46 \mathrm{GHz}$ CPU with $24 \mathrm{~GB}$ RAM. While 3D curve reconstruction (Section 4) takes about 10 to 22 seconds; 3D curve decomposition (Section 5) takes about 50 to 60 seconds for simple models (e.g., HUMAN) and a few minutes for complex models (e.g., CART). The complexity of a wire sculpture is not directly based on the number of wires it is composed of, instead it depends on the density of the wires since denser configurations result in large number of candidate 3D curves.

\section{Comparisons}

We compare our approach to PMVS (Furukawa and Ponce 2010), a state-of-the-art multi-view stereo (MVS) algorithm, and Line3D++, a recent line-based MVS algorithm (Hofer et al. 2016) developed for scene abstraction. We run each of these methods on an input image set composed of 15 views including the original 3 views we use for out method where the calibration is obtained by VisualSFM. (We run the same comparison on an input image set of 10 views and provide the results in the supplementary material.) As illustrated in Figure 8, even when using substantially more views, both approaches still suffer from noise and significant amount of missing data. Furthermore, neither point- nor individual line-based representations can capture the global topology of the wires and thus are

Table 1. We reconstruct a synthetic model from its rendering both using ground truth camera parameters (no noise) and by adding random noise to the camera parameters. We report the error as the average and maximum of the closest point distances between the ground truth and the reconstructed model in 3D. Distances are reported as a percentage of the length of the diagonal of the bounding box of synthetic model in 3D.

\begin{tabular}{c|c|c|c|c} 
& no noise & $0.1 \%$ noise & $0.2 \%$ noise & $0.3 \%$ noise \\
\hline avg & 0.17 & 0.35 & 0.54 & 0.6 \\
$\max$ & 0.98 & 1.02 & 2.06 & 2.89 \\
\hline
\end{tabular}

Table 2. For real examples, we compute a re-projection error by sampling points on the reconstructed 3D wires, projecting these samples to each input view, and measuring the distance between the projected points and the closest points sampled on the 2D curves in the view. We report the average and maximum of such re-projection errors for each input view as a percentage of the length of the diagonal of the 2D bounding box of the wiry object in the corresponding view.

\begin{tabular}{c|cc|cc|cc}
\multicolumn{2}{c}{} & \multicolumn{2}{c}{ view 1 } & \multicolumn{2}{c}{ view 2 } & \multicolumn{2}{c}{ view 3 } \\
\hline & avg & $\max$ & avg & $\max$ & avg & $\max$ \\
\hline HUMAN & 0.29 & 1.70 & 0.37 & 0.92 & 0.37 & 1.85 \\
BIKE & 0.19 & 0.94 & 0.19 & 1.36 & 0.22 & 1.65 \\
BIRD & 0.13 & 0.58 & 0.14 & 0.88 & 0.14 & 0.78 \\
ELEPHANT & 0.18 & 0.85 & 0.21 & 2.47 & 0.17 & 1.14 \\
CAT & 0.26 & 1.28 & 0.40 & 3.66 & 0.24 & 1.50 \\
FLOWER & 0.51 & 3.99 & 0.47 & 3.43 & 0.41 & 3.53 \\
HORSE & 0.24 & 1.11 & 0.24 & 2.01 & 0.21 & 1.56 \\
CART & 0.16 & 0.59 & 0.18 & 1.05 & 0.21 & 1.78 \\
TURTLE & 0.26 & 2.45 & 0.57 & 3.18 & 0.29 & 1.86 \\
\hline
\end{tabular}

not suitable for any post-processing operations. We evaluate the recent L1-medial skeleton extraction method (Huang et al. 2013) on the point clouds generated by PMVS (we include the parameters we use for this method in the supplementary material). As shown in Figure 8, due to noise and missing data in the PMVS output, this approach fails to recover a complete and accurate curve skeleton in many cases demonstrating the impracticality of utilizing similar approaches as a post-processing step.

We also run our mTSP formulation on the output of Line3D++ and observe that small variations lead to large topological errors in the final output. This demonstrates the importance of our 3D curve selection formulation (Section 4.2).

Finally, in Figure 2, we provide an example depth map of a wiry object captured from a commodity depth sensor (Microsoft Kinect). Even though we paint the originally black wire model with a diffuse paint, the sensor fails to provide any useful depth information for the thin wires that is required for surface reconstruction.

\section{Limitations}

Our method provides an effective and practical solution to a very challenging problem: we digitize wire objects from as few as $3 \mathrm{im}$ ages in the form of a global curve network readily available for post processing and fabrication. Nevertheless, there are certain limitations we would like to address in future work. Failures in extracting
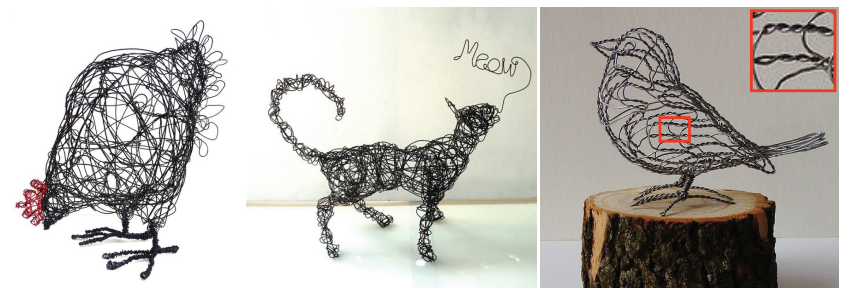

Fig. 9. Our method is not designed to handle very dense wire sculptures that are closer to surfaces instead of 1D elements.@Pinterest 
reliable 2D image curves, e.g., due to cluttered background or insufficient contrast between the object and the background, will degrade the performance of our approach like any other image-based method operating at the level of curves. We assume the camera parameters of the input images to be given and rely on surrounding texture objects to obtain this information. Significant deviations from the true camera parameters will potentially result in noisy $3 \mathrm{D}$ curve segment reconstructions and failures in the wire decomposition. A joint framework that optimizes both for the camera parameters and the 3D wires is an interesting research direction. Finally, our method is not designed to handle very dense wire sculptures such as shown in Figure 9. Such examples are closer to surfaces instead of 1D elements and other modeling paradigms (e.g., procedural modeling) would be more suitable.

\section{CONCLUSION AND FUTURE WORK}

We present an image-based reconstruction method of wire objects using as few as 3 images as input. Our method exploits unique characteristics of wire objects (simplicity - the object is composed of a few wires, and smoothness - each wire is bent smoothly) to recover the global 3D wire decomposition. We represent each wire as a curve in our reconstructions to facilitate editing and physical fabrication of the results. Combined with the power of wire sculpting, we believe that this paves the road to using wires as a rapid prototyping tool to bring creative ideas into the digital design process.

In addition to addressing the limitations of our method discussed in the previous section, there are several exciting future research directions. For our experiments, we utilized 3 input views for each example, which all together provide a full coverage of the input object. Exploring strategies for next best view selection in the context of wire objects is a promising reserch direction. Our current approach focuses on objects made by bending and connecting multiple wires. While bending is one of the most prominent techniques used in wire art, there are other techniques, e.g., twisting two wires as seen in the bird example in Figure 9 and wrapping thin wires around thicker ones. Exploring such techniques as additional priors in the reconstruction method (e.g., physical stability of the reconstructed object) is a promising direction likely to expand the scope of the objects that can be captured accurately.

\section{ACKNOWLEDGMENTS}

We thank our reviewers for their invaluable comments. We also thank Aron Monszpart, Tuanfeng Y. Wang, and Xiaoguang Han for their help, comments, and ideas. This work was partially funded by the ERC Starting Grant SmartGeometry (StG-2013-335373), the Research Grant Council of Hong Kong (HKU 717813E, 17209815, 17211017), an UCL visiting student program, and gifts from Adobe.

\section{REFERENCES}

C. Baillard, C. Schmid, A. Zisserman, and A. W. Fitzgibbon. 1999. Automatic Line Matching and 3D Reconstruction of Buildings from Multiple Views. In ISPRS Conf. on Automatic Extraction of GIS Objects from Digital Imagery. 69-80.

M. Berger, A. Tagliasacchi, L. Seversky, P. Alliez, J. Levine, A. Sharf, and C. Silva. 2014. State of the Art in Surface Reconstruction from Point Clouds. In CGF Eurographics State of the Art Reports. DOI : https://doi.org/10.2312/egst.20141040

Rikard Berthilsson, Kalle Astrom, and Anders Heyden. 2001. Reconstruction of General Curves, Using Factorization and Bundle Adjustment. IfCV 41, 3 (Feb. 2001), 171-182. DOI : https://doi.org/10.1023/A:1011104020586
Tao Chen, Zhe Zhu, Ariel Shamir, Shi-Min Hu, and Daniel Cohen-Or. 2013. 3-Sweep: Extracting Editable Objects from a Single Photo. In ACM SIGGRAPH Asia. ACM, New York, NY, USA, Article 195, 10 pages. DOI : https://doi.org/10.1145/2508363.2508378

Charlotte Delmas, Marie-Odile Berger, Erwan Kerrien, Cyril Riddell, Yves Trousset, René Anxionnat, and Serge Bracard. 2015. Three-dimensional curvilinear device reconstruction from two fluoroscopic views. In SPIE, Medical Imaging 2015: ImageGuided Procedures, Robotic Interventions, and Modeling, Vol. 9415. San Diego, CA, France, 94150F. DOI : https://doi.org/10.1117/12.2081885

R. Fabbri and B. Kimia. 2010. 3D curve sketch: Flexible curve-based stereo reconstruction and calibration. In IEEE CVPR. 1538-1545. DOI : https://doi.org/10.1109/CVPR.2010 5539787

Ricardo Fabbri and Benjamin B. Kimia. 2016. Multiview Differential Geometry of Curves. IfCV 120, 3 (Dec. 2016), 324-346. DOI : https://doi.org/10.1007/s11263-016-0912-7

Xinyi Fan, Linguang Zhang, Benedict Brown, and Szymon Rusinkiewicz. 2016. Automated View and Path Planning for Scalable Multi-object 3D Scanning. In ACM SIGGRAPH Asia. ACM, New York, NY, USA, Article 239, 13 pages. DOI : https: //doi.org/10.1145/2980179.2980225

Yasutaka Furukawa and Jean Ponce. 2010. Accurate, Dense, and Robust Multiview Stereopsis. IEEE PAMI 32, 8 (Aug. 2010), 1362-1376. DOI: https://doi.org/10.1109/ TPAMI.2009.161

M. Goesele, N. Snavely, B. Curless, H. Hoppe, and S. M. Seitz. 2007. Multi-View Stereo for Community Photo Collections. In IEEE ICCV. 1-8. DOI : https://doi.org/10.1109/ ICCV.2007.4408933

Inc. Gurobi Optimization. 2016. Gurobi Optimizer Reference Manual. (2016). http: //www.gurobi.com

R. I. Hartley and A. Zisserman. 2004. Multiple View Geometry in Computer Vision (second ed.). Cambridge University Press, ISBN: 0521540518.

Manuel Hofer, Michael Maurer, and Horst Bischof. 2014. Improving Sparse 3D Models for Man-Made Environments Using Line-Based 3D Reconstruction. In International Conference on $3 D$ Vision ( $3 D V$ ).

Manuel Hofer, Michael Maurer, and Horst Bischof. 2016. Efficient 3D scene abstraction using line segments. Computer Vision and Image Understanding (2016). DOI : https: //doi.org/10.1016/j.cviu.2016.03.017

Manuel Hofer, Andreas Wendel, and Horst Bischof. 2013. Line-based 3D reconstruction of wiry objects. In Computer Vision Winter Workshop (CVWW).

Wei Hong, Yi Ma, and Yizhou Yu. 2004. Reconstruction of 3-D Symmetric Curves from Perspective Images without Discrete Features. In ECCV. Springer Berlin Heidelberg, Berlin, Heidelberg, 533-545. DOI : https://doi.org/10.1007/978-3-540-24672-5_42

Hui Huang, Shihao Wu, Daniel Cohen-Or, Minglun Gong, Hao Zhang, Guiqing Li, and Baoquan Chen. 2013. L1-medial Skeleton of Point Cloud. In ACM SIGGRAPH. ACM, New York, NY, USA, Article 65, 8 pages. DOI : https://doi.org/10.1145/2461912. 2461913

Emmanuel Iarussi, Wilmot Li, and Adrien Bousseau. 2015. WrapIt: Computer-assisted Crafting of Wire Wrapped Jewelry. In ACM SIGGRAPH Asia. ACM, New York, NY, USA, Article 221, 8 pages. DOI : https://doi.org/10.1145/2816795.2818118

Arjun Jain, Christian Kurz, Thorsten Thormählen, and Hans-Peter Seidel. 2010. Exploiting Global Connectivity Constraints for Reconstruction of 3D Line Segment from Images. In IEEE CVPR. San Francisco, CA.

F. Kahl and J. August. 2003. Multiview reconstruction of space curves. In IEEE ICCV 1017-1024 vol.2. DOI : https://doi.org/10.1109/ICCV.2003.1238461

Imdat Kara and Tolga Bektas. 2006. Integer linear programming formulations of multiple salesman problems and its variations. European fournal of Operational Research 174, 3 (2006), 1449 - 1458. DOI : https://doi.org/10.1016/j.ejor.2005.03.008

Peter Kovesi. 2017. Peter's Functions for Computer Vision. (2017). http://www. peterkovesi.com/matlabfns/

Guo Li, Ligang Liu, Hanlin Zheng, and Niloy J. Mitra. 2010. Analysis, Reconstruction and Manipulation Using Arterial Snakes. In ACM SIGGRAPH Asia (SIGGRAPH ASIA '10). ACM, New York, NY, USA, Article 152, 10 pages. DOI : https://doi.org/10.1145/ 1866158.1866178

Tobias Martin, Juan Montes, Jean-Charles Bazin, and Tiberiu Popa. 2014. Topologyaware Reconstruction of Thin Tubular Structures. In SIGGRAPH Asia 2014 Technical Briefs (SA '14). ACM, New York, NY, USA, Article 12, 4 pages. DOI : https://doi.org/ $10.1145 / 2669024.2669035$

Eder Miguel, Mathias Lepoutre, and Bernd Bickel. 2016. Computational Design of Stable Planar-Rod Structures. ACM SIGGRAPH 35, 4 (2016).

Irina Nurutdinova and Andrew Fitzgibbon. 2015. Towards Pointless Structure from Motion: 3D Reconstruction and Camera Parameters from General 3D Curves. In IEEE ICCV. IEEE Computer Society, Washington, DC, USA, 2363-2371. DOI : https: //doi.org/10.1109/ICCV.2015.272

J.M. Ogden. 1991. Classical gold wire. Some aspects of its manufacture and use. fewellery Studies (1991), 95-105, Abb.

D. Rao, S. J. Chung, and S. Hutchinson. 2012. CurveSLAM: An approach for visionbased navigation without point features. In 2012 IEEE/RSf International Conference on Intelligent Robots and Systems. 4198-4204. DOI : https://doi.org/10.1109/IROS. 2012.6385764 
L. Robert and O. D. Faugeras. 1991. Curve-based stereo: figural continuity and curvature. In IEEE CVPR. 57-62. DOI : https://doi.org/10.1109/CVPR.1991.139661

Noah Snavely, Steven M. Seitz, and Richard Szeliski. 2006. Photo Tourism: Exploring Photo Collections in 3D. In ACM SIGGRAPH (SIGGRAPH '06). ACM, New York, NY, USA, 835-846. DOI : https://doi.org/10.1145/1179352.1141964

D. Teney and J. Piater. 2012. Sampling-Based Multiview Reconstruction without Correspondences for 3D Edges. In Int. Conf. on 3D Imaging, Modeling, Processing, Visualization Transmission. 160-167. DOI : https://doi.org/10.1109/3DIMPVT.2012.28

Inc. The MathWorks. 2016. Optimization Toolbox User's Guide. (2016). http://www. mathworks.com/access/helpdesk/help/toolbox/optim/

Anil Usumezbas, Ricardo Fabbri, and Benjamin B. Kimia. 2016. From Multiview Image Curves to 3D Drawings. In ECCV. 70-87. DOI : https://doi.org/10.1007/ 978-3-319-46493-0_5

Changchang Wu. 2011. VisualSFM: A Visual Structure from Motion System. (2011) http://ccwu.me/vsfm/

Changchang Wu, S. Agarwal, B. Curless, and S. M. Seitz. 2011a. Multicore Bundle Adjustment. In IEEE CVPR. IEEE Computer Society, Washington, DC, USA, 30573064. DOI : https://doi.org/10.1109/CVPR.2011.5995552

C. Wu, S. Agarwal, B. Curless, and S. M. Seitz. 2011b. Multicore bundle adjustment. In IEEE CVPR. 3057-3064. DOI : https://doi.org/10.1109/CVPR.2011.5995552

Shihao Wu, Wei Sun, Pinxin Long, Hui Huang, Daniel Cohen-Or, Minglun Gong, Oliver Deussen, and Baoquan Chen. 2014. Quality-driven Poisson-guided Autoscanning. In ACM SIGGRAPH Asia. ACM, New York, NY, USA, Article 203, 12 pages. DOI : https://doi.org/10.1145/2661229.2661242

Yi Jun Xiao and Y. F. Li. 2005. Optimized stereo reconstruction of free-form space curves based on a nonuniform rational B-spline model. 7. Opt. Soc. Am. A 22, 9 (Sep 2005), 1746-1762. DOI : https://doi.org/10.1364/JOSAA.22.001746

Feilong Yan, Andrei Sharf, Wenzhen Lin, Hui Huang, and Baoquan Chen. 2014. Proactive 3D Scanning of Inaccessible Parts. In ACM SIGGRAPH Asia. ACM, New York, NY, USA, Article 157, 8 pages. DOI : https://doi.org/10.1145/2601097.2601191

Kangxue Yin, Hui Huang, Hao Zhang, Minglun Gong, Daniel Cohen-Or, and Baoquan Chen. 2014. Morfit: Interactive Surface Reconstruction from Incomplete Point Clouds with Curve-driven Topology and Geometry Control. In ACM SIGGRAPH Asia. ACM, New York, NY, USA, Article 202, 12 pages. DOI : https://doi.org/10.1145/ 2661229.2661241

\section{APPENDIX}

When a particular epipolar line becomes tangent to or overlaps with a 2D curve segment, multiple intersection points close to each other on the same curve are generated. These cluttered intersections do not define meaningful disjoint curves and cause difficulty in reconstructing $3 \mathrm{D}$ curve segments in the concerned region. We propose a curve fitting strategy to handle such degenerate cases.

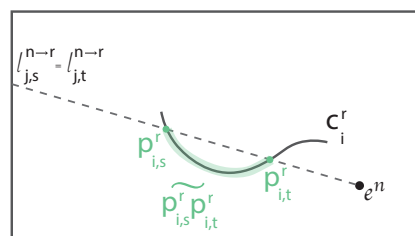

$\mathcal{I}^{\mathrm{r}}$ (reference view)

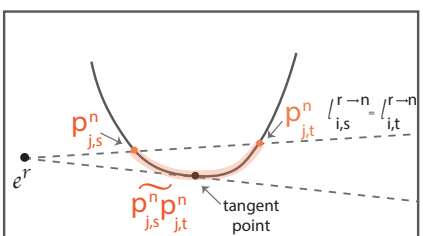

$2^{\mathrm{n}}$ (neighboring view)
Fig. 10. In case an epipolar line becomes tangent or overlaps with a 2D curve segment, we solve the resulting degenerate case by a curve fitting strategy.

When an epipolar line, $\ell$, is tangent to a smooth curve at a point of non-vanishing curvature, the part of the curve in a neighborhood of the tangent point lies entirely on the same side of $\ell$. Given the epipolar line $\ell$ that passes through a point $p$ sampled on a $2 \mathrm{D}$ curve segment, we identify $p$ as a tangent point if the $m$-nearest samples $(m=25)$ of $p$ on the curve all lie on the same side of $\ell$. This neighborhood is unstable to compute exact intersections with $\ell$.

Given a 2D curve segment $c_{i}^{r}$ in a reference view $\mathcal{I}^{r}$, assume the epipolar line, $\ell_{i, s}^{r \rightarrow n}$, of a sample point $p_{i, s}^{r}$ on $c_{i}^{r}$ in a neighboring view $\mathcal{I}^{n}$ is at a threshold distance $(\lambda=8)$ from a tangent point detected on a curve segment $c_{j}^{n}$ in $\mathcal{I}^{n}$ (see Figure 10). We denote the two intersection points between $\ell_{i, s}^{r \rightarrow n}$ and $c_{j}^{n}$ as $p_{i, s}^{n}$ and $p_{j, t}^{n}$. Moving along $c_{i}^{r}$ will yield another point $p_{i, t}^{r}$ whose epipolar line will be the same as $\ell_{i, s}^{r \rightarrow n}$. Under epipolar constraints, $p_{j, s}^{n}$ and $p_{j, t}^{n}$ have the same epipolar line in $\mathcal{I}^{r}$ and this line passes through $p_{i, s}^{r}$ and $p_{i, t}^{r}$. Thus the curve region, $\overline{p_{i, s}^{r} p_{i, t}^{r}}$ in $\mathcal{I}^{r}$ and the curve region, $\overline{p_{j, s}^{n} p_{j, t}^{n}}$ in $\mathcal{I}^{n}$ are corresponding regions.

There are two possible pairings between the matching regions $\widehat{p_{i, s}^{r} p_{i, t}^{r}}$ and $\overline{p_{j, s}^{n} p_{j, t}^{n}}$ for subsequent $3 \mathrm{D}$ curve reconstruction, that is: (i) $\left(p_{i, s}^{r}, p_{j, s}^{n}\right)$ for one end and $\left(p_{i, t}^{r}, p_{j, t}^{n}\right)$ for the other end; or (ii) $\left(p_{i, s}^{r}, p_{j, t}^{n}\right)$ for one end and $\left(p_{i, t}^{r}, p_{j, s}^{n}\right)$ for the other end. For each pairing, we first compute the $3 \mathrm{D}$ positions of the paired endpoints and then fit a polyline between these 3D endpoints that is smooth and matches the two image observations as in Equation 9. We keep both of the results as part of the candidate 3D curve segment set.

If an epipolar line is tangent to a curve at an inflection point, we detect the unstable neighborhood by checking if the $m$-nearest points of the intersection point lie within a narrow strip centered around the epipolar line. The remaining curve region process remains unchanged. 